\title{
Mixed-Criticality Scheduling with I/O
}

\author{
Eric Missimer, Katherine Zhao and Richard West \\ Computer Science Department \\ Boston University \\ Boston, MA 02215 \\ Email: \{missimer,kzhao,richwest\}@cs.bu.edu
}

\begin{abstract}
This paper addresses the problem of scheduling tasks with different criticality levels in the presence of $I / O$ requests. In mixed-criticality scheduling, higher criticality tasks are given precedence over those of lower criticality when it is impossible to guarantee the schedulability of all tasks. While mixed-criticality scheduling has gained attention in recent years, most approaches typically assume a periodic task model. This assumption does not always hold in practice, especially for realtime and embedded systems that perform I/O. In prior work, we developed a scheduling technique in the Quest real-time operating system, which integrates the time-budgeted management of I/O operations with Sporadic Server scheduling of tasks. This paper extends our previous scheduling approach with support for mixed-criticality tasks and $I / O$ requests on the same processing core. Results show that in a real implementation the mixedcriticality scheduling method introduced in this paper outperforms a scheduling approach consisting of only Sporadic Servers.
\end{abstract}

\section{INTRODUCTION}

Mixed-criticality scheduling orders the execution of tasks of different criticality levels. Criticality levels are based on the consequences of a task violating its timing requirements, or failing to function as specified. For example, DO-178B is a software certification used in avionics, which specifies several assurance levels in the face of software failures. These assurance levels range from catastrophic (e.g., could cause a plane crash) to non-critical when they have little or no impact on aircraft safety or overall operation. Mixed-criticality scheduling was first introduced by Vestal (2007) [1]. Later, Baruah, Burns and Davis (2011) [2] introduced Adaptive Mixed-Criticality (AMC) scheduling. The work presented in this paper builds upon AMC to extend it for use in systems where tasks make I/O requests. This is the first paper to address the issue of $\mathrm{I} / \mathrm{O}$ scheduling in an Adaptive MixedCriticality scenario. Our approach to AMC with I/O is based on experience with our in-house real-time operating system, called Quest [3].

Quest can be configured to have two privilege levels, with the more privileged kernel being separated from a less privileged user-space. This is similar to traditional operating systems such as Linux. In contrast, an alternative system configuration, called Quest-V, supports three privilege levels. The third privilege level in Quest-V is more trusted than the kernel, and operates as a lightweight virtual machine monitor, or hypervisor. Unlike with traditional virtual machine systems, Quest-V uses its most trusted privilege level to partition resources amongst (guest) sandbox domains. Each sandbox domain then manages its own resources independently and in isolation of other sandbox domains, without recourse to a hypervisor. This leads to a far more efficient design, where virtualization overheads are almost entirely eliminated. It has been shown in prior work that it is possible to dedicate separate tasks of different criticality levels to different sandboxes in Quest-V [4]. This is demonstrated in Figure 1, Note that each sandbox has a different criticality level with level 0 being the least critical. However, Quest-V has thus far not considered tasks of different criticality levels within the same sandbox and, hence, for scheduling on the same (shared) processor cores. In this paper, we show how to integrate the processing of I/O events and tasks in an Adaptive Mixed-Criticality [2] scheduling framework built within the Quest kernel. We extend Quest with support for mode changes between different criticality levels. This enables components of different critically levels to coexist in a single Quest- $\mathrm{V}$ sandbox or in a traditional Quest system, as depicted in Figure 2.

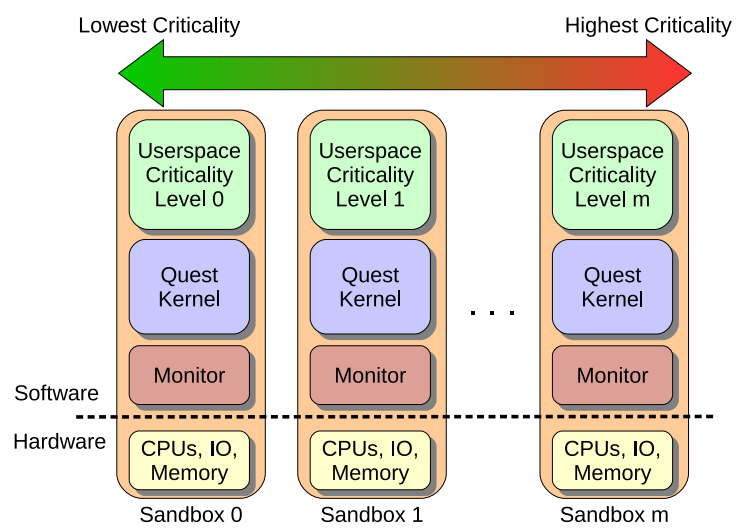

Fig. 1: Mixed-Criticality Levels Across Separate Quest-V Sandboxes

Previous mixed-criticality analysis assumes that all jobs in the system are scheduled under the same policy, typically as periodic tasks. However, as previously shown by Danish, Li and West [5], using the same scheduling policy for both task threads and bottom half interrupt handlers 1 results in lower I/O performance and larger overheads. Specifically, the authors

\footnotetext{
${ }^{1}$ We use the Linux terminology, where the top half is the non-deferrable work that runs in interrupt context, and the bottom half is the deferrable work executed in a thread context after the top half.
} 


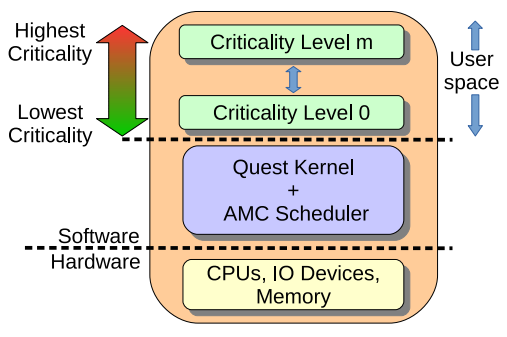

Fig. 2: Quest Support for Mixed-Criticality Scheduling

compared the Sporadic Server (SS) [6] model for both main threads and bottom half interrupt handlers to using Sporadic Servers for main threads and Priority Inheritance Bandwidthpreserving Servers (PIBS) for bottom half threads. The results showed that by using PIBS for interrupt bottom half threads, the scheduling overheads are reduced and I/O performance is increased. The details of PIBS will be discussed in Section $\amalg$

The contributions of this paper include a mixed-criticality analysis assuming threads are scheduled using either the Sporadic Server or PIBS scheduling model. It is shown that while a system of Sporadic Servers and PIBS has a slightly lower schedulability than a system of only Sporadic Servers from a theoretical point of view, in practice a real implementation of both scheduling policies results in Sporadic Server and PIBS outperforming a system of only Sporadic Servers.

The rest of the paper is organized as follows. Section $\square$ provides the necessary background information on Sporadic Servers and PIBS and introduces a response time analysis for them. Next, Section III briefly discusses the Adaptive Mixed-Criticality (AMC) model. Section IV contains the AMC scheduling analysis for a system of Sporadic and Priority Inheritance Bandwidth Preserving Servers. Section V discusses the implementation details of our AMC approach in the Quest operating system. Section VI discusses experimental results, while related work is described in Section VII Finally, conclusions are discussed in Section VIII

\section{SPORADIC SERVER AND PIBS}

Sporadic Servers (SS) [6] and Priority Inheritance Bandwidth-preserving Servers (PIBS) [5] are the two scheduling models used in the Quest real-time operating system [3]. Sporadic Servers are specified using a budget capacity, $C$, and period $T$. By default, the Sporadic Server with the smallest period is given highest priority, which follows the rate-monotonic policy [7]. The main tasks in Quest run on Sporadic Servers, thereby guaranteeing them a minimum share of CPU time every real-time period. Replenishment lists are used to track the consumption of CPU time and when it is eligible to be re-applied to the corresponding server.

PIBS uses a much simpler scheduling method which is more appropriate for the short execution times associated with interrupt bottom half threads. A PIBS is specified by a utilization, $U$. A PIBS always runs on behalf of a Sporadic Server and inherits both the priority and period of the Sporadic Server. For example, the PIBS running in response to a device interrupt would run on behalf of the Sporadic Server that requested the I/O action to be performed. The capacity of a PIBS is calculated as $C=U \times T$, where $T$ is the period of the Sporadic Server.

As with Sporadic Servers, PIBS uses replenishments but instead of a list there is only a single replenishment. When a PIBS has executed $C_{\text {actual }}$, its next replenishment is set to $t+C_{\text {actual }} / U$, where $t$ is the time the PIBS started its most recent execution. A PIBS cannot execute again until the next replenishment time regardless of whether it has utilized its entire budget or not. Since a PIBS uses only one replenishment value rather than a list, it is beneficial for scheduling shortlived interrupt service routines that would otherwise fragment a Sporadic Server's budget into many small replenishments. The replenishment method of a PIBS limits its maximum utilization within any sliding window of size $T$ to $(2-U) U$. This occurs when the PIBS first runs for $C_{1}=U(T-U T)$ and then again for $C_{2}=U T$. This is demonstrated in Figure 3 .

$$
\begin{aligned}
\frac{C_{1}+C_{2}}{T} & =\frac{\left(T^{\prime} * U\right)+C_{2}}{T} \\
& =\frac{\left(T-C_{2}\right) * U+C_{2}}{T} \\
& =\frac{\left(C_{2} / U-C_{2}\right) * U+C_{2}}{C_{2} / U} \\
& =(2-U) U
\end{aligned}
$$

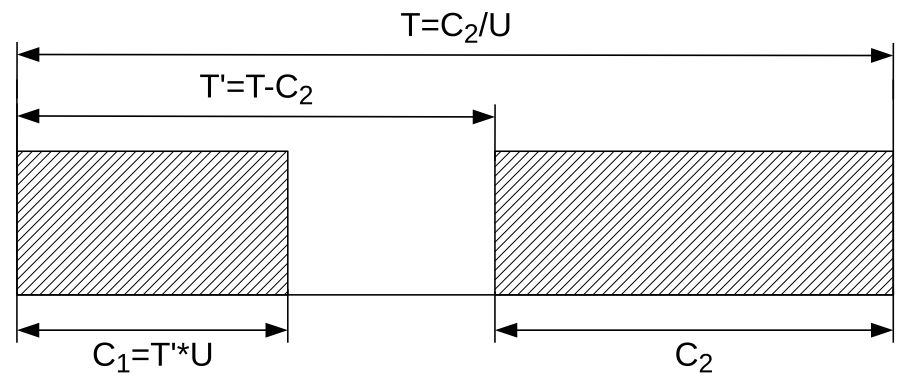

Fig. 3: PIBS Server Utilization

The interaction between Sporadic Servers and PIBS is depicted in Figure 4 First, the Sporadic Server initiates an I/O related system call (Step 1). The system call invokes the associated device driver, which programs the device (Step 2). The device will eventually initiate an interrupt which is handled by the top half interrupt handler (Step 3). The top half interrupt handler will acknowledge the interrupt and wake up one of the PIBS to handle the majority of the work associated with the interrupt (Step 4). Note that although the figure shows PIBS run at kernel-level, they could just as well be associated with user-space threads if the system granted such privileges. Finally, after a PIBS finishes executing it will wake up the corresponding Sporadic Server, assuming the Sporadic Server was blocked on an I/O request (Step 5). A more detailed description of the scheduling of PIBS and Sporadic Servers can be found in prior work (2011) [5]. 


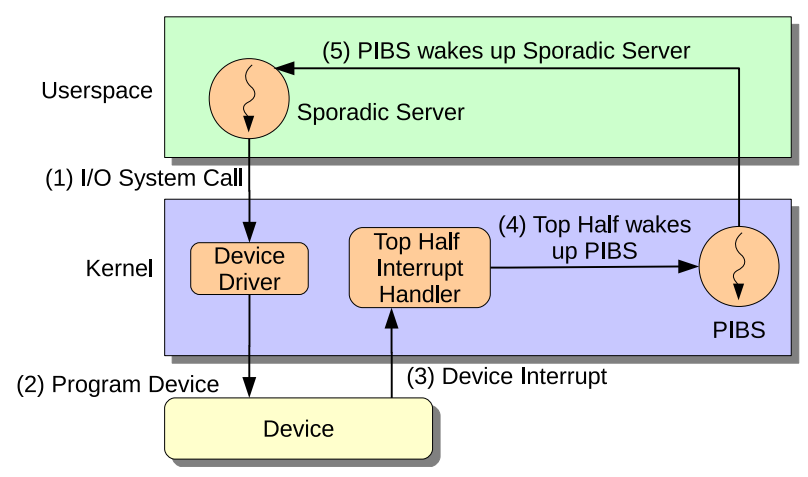

Fig. 4: Sporadic Server and PIBS Interaction

The advantages of using PIBS for bottom half interrupt handling include lower scheduling overhead and no delayed execution due to replenishment list fragmentation. The short execution time of bottom half interrupt handlers can cause a Sporadic Server to complete execution before exhausting its available capacity. This leads to a fragmented replenishment list. In practice, the lists are finite in length because of memory constraints and to limit scheduling overhead. When a replenishment list is full, items are merged to make space for new replenishments. This causes the available budget to be deferred [8], and the effective utilization of the Sporadic Server can drop below its specified value. This in turn results in deadlines being missed. In contrast, PIBS have only a single replenishment list item and a different policy for how the replenishment is posted, which prevents a drop in their effective utilization and lower scheduling overhead.

Figure 5 shows an example of replenishment list fragmentation. The first task, $\tau_{1}$, begins execution at time $t=0$ and continues execution for eight time units. $\tau_{1}$ utilized its entire capacity at $t=8$ so a single single replenishment item is posted for 8 time units of capacity at time $t=16$. The replenishment is posted at $t=16$ because $\tau_{1}$ started execution at time $t=0$ and has a period of 16. Right at the completion of its execution $\tau_{1}$ initiates an I/O related event, e.g. a read. Suppose this I/O event causes four interrupts to occur. Each interrupt initiates a bottom half thread that takes one time unit of computation to complete. $\tau_{1}$ will require all four bottom half interrupt handlers to complete execution before it can continue execution, e.g. it is blocking on the read. $\tau_{2}$ is the task responsible for handling these bottom half interrupt handlers. The first interrupt occurs at time $t=9$ and is immediately handled by $\tau_{2}$. Note that at time $t=9$ the time of the head replenishment list item is updated from zero to nine. This is to ensure that when a replenishment item is posted after the task blocks or depletes its budget the replenishment item is posted at the correct time. Once $\tau_{2}$ completes execution of the bottom half interrupt handler it blocks as it waits for another I/O interrupt to occur.

When $\tau_{2}$ blocks it posts a replenishment item for the capacity that it used. Since it used 1 time unit of capacity and started executing at time $t=9$ a replenishment item of 1 time unit is posted at time $t=25$. At time $t=11$ another interrupt occurs, waking up $\tau_{2}$ for another time unit. The time of the first replenishment list item is updated to 11 to reflect that the Sporadic Server started execution at time $t=11$. After handling the bottom half interrupt handler, another replenishment item for one time unit is posted, this time at time $t=27$. When the third interrupt occurs $\tau_{2}$ again executes for 1 time unit. However, when $\tau_{2}$ attempts to post a replenishment item for the one unit of capacity used it cannot since its replenishment list is full 2 In order to ensure that $\tau_{2}$ does not adversely affect other running tasks, its remaining capacity of one time unit is merged with the next replenishment list item, which in this example is at $t=25$. This results in the available capacity for $\tau_{2}$ being zero, leaving it unable to immediately handle the interrupt that occurs at time $t=15$. Instead, the execution of the interrupt is delayed and completes only at time $t=26$. Meanwhile, $\tau_{1}$, which had the capacity to execute at time $t=16$, is blocking waiting for completion of the fourth interrupt handler. $\tau_{1}$ begins execution at time $t=26$ but that leaves only six time units until the deadline at time $t=32$, instead of the eight required to complete execution.

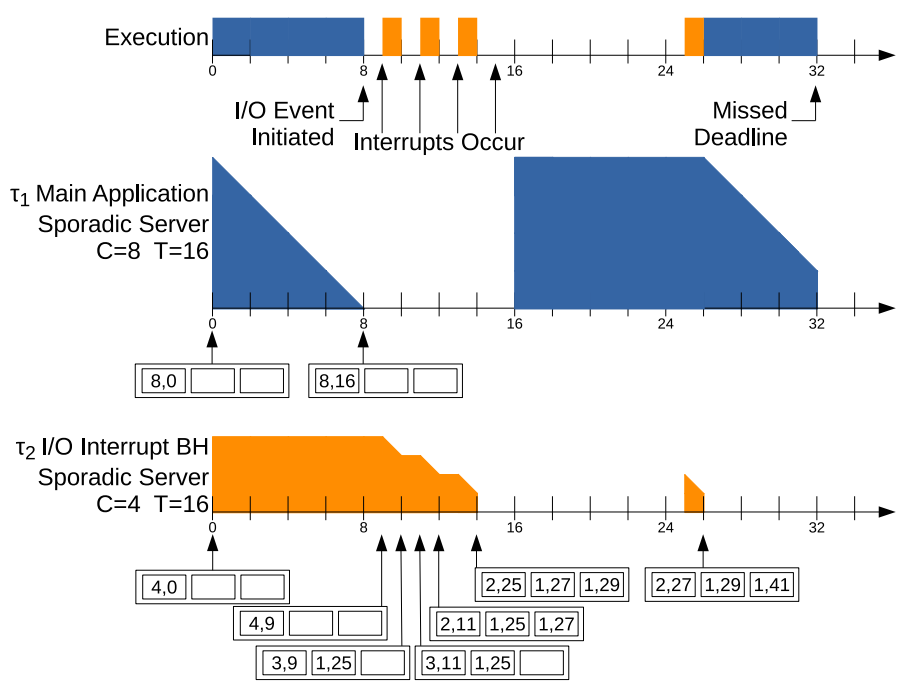

Fig. 5: Example Task and I/O Scheduling using Sporadic Servers

Figure 6 shows a similar scheduling scenario. However, this time the interrupt bottom halves are handled by a PIBS. As with the previous scenario, $\tau_{1}$ initiates an I/O related event at time $t=8$ and blocks until the completion of the event. The first interrupt occurs at time $t=9$ and is immediately handled by PIBS. As with the Sporadic Server, the time in the replenishment list item is updated to reflect when the PIBS started execution. Once the event is handled, the PIBS posts a single replenishment item at time $t=13$. This is because $\tau_{2}$ is running on behalf of $\tau_{1}$ so it inherits both the priority and period of $\tau_{1}$. Since $\tau_{2}$ executed for only $25 \%$ of its available four time units of capacity the replenishment is posted $25 \%$ of its period from when it started execution. The second interrupt

\footnotetext{
${ }^{2}$ For the sake of this example the replenishment list size is three. In practice, a larger replenishment list size would be chosen but, regardless, fragmentation and capacity postponement can occur [5].
} 
occurs at time $t=11$ but its execution is deferred until $\tau_{2}$ has available capacity. At time $t=13$ the third interrupt arrives and $\tau_{2}$ has the capacity to handle both it and the previous interrupt. Finally, the fourth interrupt arrives at time $t=15$, which can also be handled by $\tau_{2}$. Since $\tau_{2}$ has executed for $75 \%$ of its available capacity a replenishment is posted twelve time units after it started execution, at $t=25$. This permits $\tau_{1}$ to continue execution at time $t=16$. The pattern then repeats itself. This simple example demonstrates the advantages of PIBS for bottom half threads compared to Sporadic Servers. Finally, note that even if the replenishment list in the first example had been long enough to avoid the delayed budget, the Sporadic Server would have experienced twice as much context switching overhead compared to the equivalent PIBS.
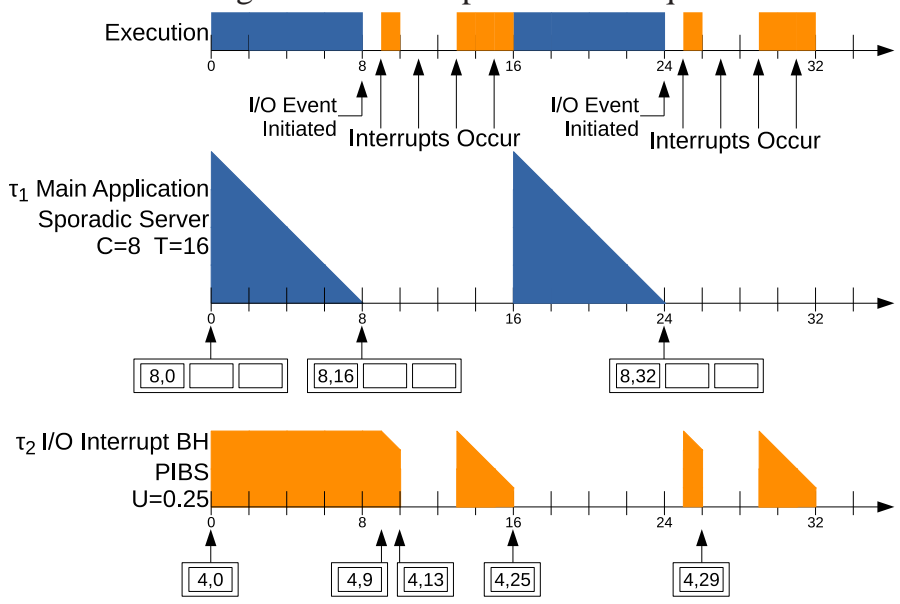

Fig. 6: Example Task and I/O Scheduling using Sporadic Servers \& PIBS

Note that in the first example, if a different policy for handling a full Sporadic Server replenishment list had been chosen, $\tau_{2}$ might have completed in time for $\tau_{1}$ to finish before its deadline. For example, if the later replenishment items were merged instead of the head replenishment item, $\tau_{2}$ would have had one remaining time unit of capacity to handle the last bottom half interrupt handler. However, as more interrupts occur, this temporary fix will no longer work as more capacity is delayed further in time.

\section{A. Response Time Analysis for SS and PIBS}

In order to perform an Adaptive Mixed-Criticality analysis for a combined Sporadic Server and PIBS system, the response time analysis equation of the system must be derived. First, under the assumption that a Sporadic Server can be treated as an equivalent periodic task [6], the response time equation for task $\tau_{i}$ in a system of only Sporadic Servers is the following:

$$
R_{i}=C_{i}+\sum_{\tau_{j} \in \mathbf{h p}(i)}\left\lceil\frac{R_{i}}{T_{j}}\right\rceil C_{j}
$$

where hp $(i)$ is the set of tasks of equal or higher priority than task $\tau_{i}$. Second, due to the worst-case phasing of a combined system of PIBS and Sporadic Servers, a PIBS utilization bound of $(2-U) U$ cannot repeatedly occur. The worst case phasing can result in at most an additional capacity (i.e., execution time) of $\left(T_{q}-T_{q} U_{k}\right) U_{k}$ for PIBS $\tau_{k}$ assigned to the Sporadic Server $\tau_{q}$. This is only possible if PIBS blocks waiting on I/O before consuming its full budget capacity. Therefore, a tighter upper-bound on the interference a PIBS can cause is:

$$
\begin{aligned}
I_{k}^{q}(t) & =\left(T_{q}-T_{q} U_{k}\right) U_{k}+\left\lceil\frac{t}{T_{q}}\right\rceil T_{q} U_{k} \\
& =\left(1-U_{k}\right) T_{q} U_{k}+\left\lceil\frac{t}{T_{q}}\right\rceil T_{q} U_{k} \\
& =\left(1+\left\lceil\frac{t}{T_{q}}\right\rceil-U_{k}\right) T_{q} U_{k}
\end{aligned}
$$

This can be incorporated into the response time analysis of Sporadic Server $\tau_{i}$, in a system consisting of both Sporadic Servers and PIBS, in the following way:

$$
\begin{aligned}
R_{i}=C_{i} & +\sum_{\tau_{j} \in \mathbf{h p}(i)}\left\{\left\lceil\frac{R_{i}}{T_{j}}\right\rceil C_{j}\right\} \\
& +\sum_{\tau_{k} \in \mathbf{p s}} \max _{\tau_{q} \in \mathbf{h i p}(i)}\left\{I_{k}^{q}\left(R_{i}\right)\right\}
\end{aligned}
$$

Where ps is the set of all PIBS and hip $(i)=\mathbf{h p}(i) \cup\left\{\tau_{i}\right\}$, i.e. the set containing $\tau_{i}$ and all tasks with equal or higher priority than task $\tau_{i}$. This is necessary as the PIBS can be running on behalf of task $\tau_{i}$. In general, there is no apriori knowledge about which PIBS runs for which Sporadic Server. Therefore, the Sporadic Server, $\tau_{q}$ that maximizes the interference caused by the PIBS must be considered. If such a-priori knowledge existed, it could be used to reduce the possible set of Sporadic Servers on behalf of which a PIBS could be executing. However, without such knowledge all possible Sporadic Server tasks of equal or higher priority must be considered.

The response time analysis for a PIBS is therefore dependent on the associated Sporadic Server. The response time analysis for PIBS $\tau_{p}$ when assigned to Sporadic Server $\tau_{s}$ is:

$$
\begin{aligned}
{ }_{s} R_{p}= & \left(2-U_{p}\right) U_{p} T_{s}+\sum_{\tau_{j} \in \mathbf{h i p}(s)}\left\{\left\lceil\frac{{ }_{s} R_{p}}{T_{j}}\right\rceil C_{j}\right\} \\
& +\sum_{\tau_{k} \in \mathbf{p s} \backslash\left\{\tau_{p}\right\}} \max _{\tau_{q} \in \mathbf{h i p}(s)}\left\{I_{k}^{q}\left({ }_{s} R_{p}\right)\right\}
\end{aligned}
$$

Note that $\left(2-U_{p}\right) U_{p} T_{s}$ is the maximum execution time of the PIBS over a time window of $T_{s}$, i.e. $I_{p}^{s}\left(T_{s}\right)=\left(2-U_{p}\right) U_{p} T_{s}$. Besides the first terms differing, Equation 2 differs from Equation 1 in that hip $(s)$ is used instead of $\mathbf{h p}(s)$ for the set of Sporadic Servers. This is because Sporadic Server $\tau_{s}$ must be included as it has an equal priority to PIBS $\tau_{p}$ when $\tau_{p}$ is running on behalf of $\tau_{s}$. Also, the summation over all PIBS does not include PIBS $\tau_{p}$ when determining its response time. If ${ }_{s} R_{p} \leq T_{s}$, for each and every Sporadic Server $\tau_{s}$ that $\tau_{p}$ can be assigned to, then $\tau_{p}$ will never miss a deadline.

\section{BACKGROUND: AMC SCHEDULING}

This section will provide the necessary background information on Adaptive Mixed-Criticality (AMC) scheduling [2] to 
understand the analysis in SectionIV A more detailed analysis can be found in Baruah (2011) [2].

In AMC, a task $\tau_{i}$ is defined by its period, deadline, a vector of computation times and a criticality level, $\left(T_{i}, D_{i}, \vec{C}_{i}, L_{i}\right)$. In the simplest case, $L_{i} \in\{\mathrm{LO}, \mathrm{HI}\}$, i.e. there are two criticality levels LO and HI where HI $>$ LO. For tasks for which $L=\mathrm{LO}, C(H I)$ is not defined as there are no HI-criticality versions of these tasks to execute. For HI-criticality tasks $C(\mathrm{HI}) \geq C(\mathrm{LO})$. The system also has a criticality level and it initially starts in the LO-criticality mode. While running in the LO-criticality mode, both LO- and HI-criticality tasks execute, and while running in HI-criticality mode, only HI-criticality tasks execute. If a high criticality task exhausts its $C(L O)$ before finishing its current job, the system switches into the HI-criticality mode and suspends all LO-criticality tasks. This requires a signaling mechanism available to tasks to signal that they have completed execution of a specific job instance.

The schedulability test for AMC consists of three parts: 1) the schedulability of the tasks when the system is in the LOcriticality state, 2) the schedulability of the tasks when the system is in the HI-criticality state and 3) the schedulability of the tasks during the mode change from LO-criticality to HI-criticality. The first two are simple and can be handled with the traditional response time analysis, taking into account the appropriate set of tasks and worst case execution times. Specifically, the response time analysis for each task $\tau_{i}$ when the system is in the LO-criticality state is:

$$
R_{i}^{\mathrm{LO}}=C_{i}(\mathrm{LO})+\sum_{\tau_{j} \in \mathbf{h p}(i)}\left\lceil\frac{R_{i}^{\mathrm{LO}}}{T_{j}}\right\rceil C_{j}(\mathrm{LO})
$$

and the response time analysis for the HI-criticality state is:

$$
R_{i}^{\mathrm{HI}}=C_{i}(\mathrm{HI})+\sum_{\tau_{j} \in \mathbf{h p H}(i)}\left\lceil\frac{R_{i}^{\mathrm{HI}}}{T_{j}}\right\rceil C_{j}(\mathrm{HI})
$$

where $\mathbf{h p H}(i)$ is the set of all high-criticality tasks with a priority higher than or equal to that of task $\tau_{i}$.

What remains is whether all HI-criticality tasks will meet their deadlines during the mode change from LO-criticality to HI-criticality. Baruah, Burns and Davis provided two sufficient but not complete scheduling tests for the criticality mode, i.e. the tests will not admit task sets that are not schedulable but may reject task sets that are schedulable. The first is AMCrtb (response time bound) which derives a new response time analysis equation for the mode change. The second is AMCmax which derives an expression for the maximum interference a HI-criticality task can experience during the mode change. AMC-max iterates over all possible points in time where the interference could increase, taking the maximum of these points. AMC-max is more computationally expensive than AMC-rtb but dominates AMC-rtb by permitting certain task sets that AMC-rtb rejects, and accepting any task set that AMC-rtb accepts. Both tests use Audsley's priority-assignment algorithm [9], as priorities that are inversely related to period are not optimal for AMC [1], [2].
In this paper, we focus on the use of AMC-rtb for response time analysis of a system with Sporadic Servers and PIBS. This is because of the added expense incurred by AMC-max, which must iterate over all time points when LO-criticality tasks are released.

The AMC-rtb analysis starts with a modified form of the traditional periodic response time analysis:

$$
R_{i}^{*}=C_{i}+\sum_{\tau_{j} \in \mathbf{h p}(i)}\left\lceil\frac{R_{i}^{*}}{T_{j}}\right\rceil C_{j}\left(\min \left(L_{i}, L_{j}\right)\right)
$$

Where $\min \left(L_{i}, L_{j}\right)$ returns the lowest criticality level passed to it, e.g. in the case of a dual-criticality level system, HI is only returned if both arguments are HI. The use of min implies that we only consider criticality levels equal to or less than the criticality level of $\tau_{i}$. If we divide the higher priority tasks by criticality level, we obtain the following:

$$
\begin{aligned}
R_{i}^{*}=C_{i} & +\sum_{\tau_{j} \in \mathbf{h p H}(i)}\left\lceil\frac{R_{i}^{*}}{T_{j}}\right\rceil C_{j}\left(\min \left(L_{i}, L_{j}\right)\right) \\
& +\sum_{\tau_{j} \in \mathbf{h p L}(i)}\left\lceil\frac{R_{i}^{*}}{T_{j}}\right\rceil C_{j}(\mathrm{LO})
\end{aligned}
$$

Where $\mathbf{h p L}(i)$ is the set of all LO-criticality tasks with a priority higher than or equal to the priority of task $\tau_{i}$. The min in the third term is replaced with $\mathrm{LO}$ as we know $L_{j}=\mathrm{LO}$. Since we are only concerned with high priority tasks after the mode change, i.e. $L_{i}=\mathrm{HI}$, Equation 4 becomes:

$$
\begin{aligned}
R_{i}^{*}=C_{i}(\mathrm{HI}) & +\sum_{\tau_{j} \in \mathbf{h p H}(i)}\left\lceil\frac{R_{i}^{*}}{T_{j}}\right\rceil C_{j}(\mathrm{HI}) \\
& +\sum_{\tau_{j} \in \mathbf{h p L}(i)}\left\lceil\frac{R_{i}^{*}}{T_{j}}\right\rceil C_{j}(\mathrm{LO})
\end{aligned}
$$

Finally, the response time bound can be tightened even further by recognizing that LO-criticality tasks can only interfere with HI-criticality tasks before the change has occurred. With this observation the final AMC response time bound equation is:

$$
\begin{aligned}
R_{i}^{*}=C_{i}(\mathrm{HI}) & +\sum_{\tau_{j} \in \mathbf{h p H}(i)}\left\lceil\frac{R_{i}^{*}}{T_{j}}\right\rceil C_{j}(\mathrm{HI}) \\
& +\sum_{\tau_{j} \in \mathbf{h p L}(i)}\left\lceil\frac{R_{i}^{\mathrm{LO}}}{T_{j}}\right\rceil C_{j}(\mathrm{LO})
\end{aligned}
$$

\section{A. LO-criticality tasks running in the HI-criticality mode}

Burns and Baruah [10] provide an extension to AMC that permits lower criticality tasks to continue execution in the HIcriticality state. This extension is used in our AMC model with support for $\mathrm{I} / \mathrm{O}$, which is briefly summarized as follows:

If LO-criticality tasks are allowed to continue execution in the HI-criticality mode at a lower capacity, the following is 
the response time for a HI-criticality task $\tau_{i}$ :

$$
\begin{aligned}
R_{i}^{*}=C_{i} & +\sum_{\tau_{j} \in \mathbf{h p H}(i)}\left\lceil\frac{R_{i}^{*}}{T_{j}}\right\rceil C_{j}(\mathrm{HI}) \\
& +\sum_{\tau_{j} \in \mathbf{h p L}(i)}\left\lceil\frac{R_{i}^{\mathrm{LO}}}{T_{j}}\right\rceil C_{j}(\mathrm{LO}) \\
& +\sum_{\tau_{j} \in \mathbf{h p L}(i)}\left(\left\lceil\frac{R_{i}^{*}}{T_{j}}\right\rceil-\left\lceil\frac{R_{i}^{\mathrm{LO}}}{T_{j}}\right\rceil\right) C_{j}(\mathrm{HI})
\end{aligned}
$$

The final term in Equation 7 expresses the maximum number of times the LO-criticality task will be released multiplied by

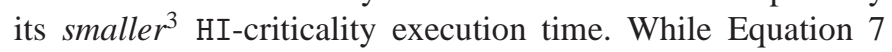
also applies to LO-criticality tasks that continue running after the mode change, a tighter bound is possible. Specifically, if a LO-criticality task has already run for $C(\mathrm{HI})$ before the mode change then it has met its HI-criticality requirement. Therefore, $R_{i}^{\mathrm{LO}}$ can be replaced with a smaller value for LOcriticality tasks. To this end $R_{i}^{\mathrm{LO} *}$ is defined as the following:

$$
\begin{array}{r}
R_{i}^{\mathrm{LO} *}=\min \left(C_{i}(\mathrm{LO}), C_{i}(\mathrm{HI})\right)+ \\
\sum_{\tau_{j} \in \mathbf{h p}(i)}\left\lceil\frac{R_{i}^{\mathrm{LO} *}}{T_{j}}\right\rceil C_{j}(\mathrm{LO})
\end{array}
$$

Note that $R_{i}^{\mathrm{LO} *}=R_{i}^{\mathrm{LO}}$ if $L_{i}=\mathrm{HI}$ and $R_{i}^{\mathrm{LO} *} \leq R_{i}^{\mathrm{LO}}$ if $L_{i}=\mathrm{LO}$, as LO-criticality tasks will have a smaller capacity in the HIcriticality mode. Therefore, Equation 7 can be replaced with the following more general equation that is tighter for LOcriticality tasks:

$$
\begin{aligned}
R_{i}^{*}= & C_{i}+\sum_{\tau_{j} \in \mathbf{h p H}(i)}\left\lceil\frac{R_{i}^{*}}{T_{j}}\right\rceil C_{j}(\mathrm{HI}) \\
& +\sum_{\tau_{j} \in \mathbf{h p L}(i)}\left\lceil\frac{R_{i}^{\mathrm{LO} *}}{T_{j}}\right\rceil C_{j}(\mathrm{LO}) \\
& +\sum_{\tau_{j} \in \mathbf{h p L}(i)}\left(\left\lceil\frac{R_{i}^{*}}{T_{j}}\right\rceil-\left\lceil\frac{R_{i}^{\mathrm{LO} *}}{T_{j}}\right\rceil\right) C_{j}(\mathrm{HI})
\end{aligned}
$$

In Section IV we will use both AMC models described in this section to derive an AMC model for a system that includes Priority Inheritance Bandwidth-Preserving Servers.

\section{AMC SPORADIC SERVER AND PIBS SCHEDULING}

This section describes the system model for I/O Adaptive Mixed-Criticality (IO-AMC), comprising both Sporadic Servers and Priority Inheritance Bandwidth-Preserving Servers (PIBS). IO-AMC focuses on the scheduling of I/O events and application threads in a mixed-criticality setting. Based on the IO-AMC model, we will derive a response time bound, IOAMC-rtb, for Sporadic Servers and PIBS.

\footnotetext{
${ }^{3}$ For LO-criticality tasks that can execute in HI-criticality mode, $C(\mathrm{LO})>C(\mathrm{HI})$, whereas for HI-criticality tasks $C(\mathrm{HI}) \geq C(\mathrm{LO})$.
}

\section{A. I/O Adaptive Mixed-Criticality Model}

Sporadic Servers follow a similar model to the original AMC model. A Sporadic Server task $\tau_{i}$ is assigned a criticality level $L_{i} \in\{\mathrm{LO}, \mathrm{HI}\}$, a period $T_{i}$ and a vector of capacities $\vec{C}_{i}$. The deadline is assumed to be equal to the period. If $L_{i}=\mathrm{LO}$, $\tau_{i}$ only runs while the system is in the LO-criticality mode and therefore only $C(\mathrm{LO})$ is defined. For HI-criticality tasks both $C(\mathrm{LO})$ and $C(\mathrm{HI})$ are defined and $C(\mathrm{HI}) \geq C(\mathrm{LO})$.

For PIBS, an I/O task $\tau_{k}$ is again assigned to either the LO or HI criticality level; $L_{k} \in\{\mathrm{LO}, \mathrm{HI}\}$. As previously discussed, PIBS are only defined by a utilization $U_{k}$. The period, deadline and priority for a PIBS is inherited from the Sporadic Server for which it is performing a task. For IO-AMC, this definition is extended and each PIBS is defined by a vector of utilizations $\vec{U}_{k}$. If $\tau_{k}$ is a LO-criticality PIBS, i.e. $L_{k}=\mathrm{LO}$, then $U_{k}(\mathrm{LO})>U_{k}(\mathrm{HI})$ and if $L_{k}=\mathrm{HI}$ then $U_{k}(\mathrm{LO}) \leq U_{k}(\mathrm{HI})$. This definition allows LO-criticality PIBS to continue execution after the switch to HI-criticality. This model allows users to assign criticality levels to $\mathrm{I} / \mathrm{O}$ devices indirectly by assigning criticality levels to the PIBS that execute in response to the I/O device.

With the typical AMC model now augmented to consider PIBS we can now derive a new admissions test for IO-AMC. First, the PIBS interference equation introduced in Section II is modified to incorporate criticality levels:

$$
I_{k}^{q}(t, L)=\left(1+\left\lceil\frac{t}{T_{q}}\right\rceil-U_{k}(L)\right) T_{q} U_{k}(L)
$$

As before, there are three conditions that must be considered: (1) the LO-criticality steady state, (2) the HI-criticality steady state, and (3) the change from LO-criticality to HIcriticality. The steady states are again simple and are merely extensions of the non-mixed-criticality response time bounds. For Sporadic Server tasks the steady state equations are:

$$
\begin{aligned}
R_{i}^{\mathrm{LO}}=C_{i}(\mathrm{LO}) & +\sum_{\tau_{j} \in \mathbf{h p}(i)}\left\{\left\lceil\frac{R_{i}^{\mathrm{LO}}}{T_{j}}\right\rceil C_{j}(\mathrm{LO})\right\} \\
& +\sum_{\tau_{k} \in \mathbf{p s}} \max _{\tau_{q} \in \mathbf{h i p}(i)}\left\{I_{k}^{q}\left(R_{i}^{\mathrm{LO}}, \mathrm{LO}\right)\right\} \\
R_{i}^{\mathrm{HI}}=C_{i}(\mathrm{HI}) & +\sum_{\tau_{j} \in \mathbf{h p H}(i)}\left\{\left\lceil\frac{R_{i}^{\mathrm{HI}}}{T_{j}}\right\rceil C_{j}(\mathrm{HI})\right\} \\
& +\sum_{\tau_{k} \in \mathbf{p s}} \max _{\tau_{q} \in \mathbf{h i p H}(i)}\left\{I_{k}^{q}\left(R_{i}^{\mathrm{HI}}, \mathrm{HI}\right)\right\}
\end{aligned}
$$

where $\operatorname{hipH}(i)=\mathbf{h p H}(i) \cup\left\{\tau_{i}\right\}$, i.e. it is the set of all HIcriticality tasks of higher or equal priority than task $\tau_{i}$, plus task $\tau_{i}$ itself. For PIBS task $\tau_{p}$, running on behalf of Sporadic Server task $\tau_{s}$, the steady state equations are:

$$
\begin{aligned}
{ }_{s} R_{p}^{\mathrm{LO}}= & \left(2-U_{p}(\mathrm{LO})\right) U_{p}(\mathrm{LO}) T_{s} \\
& +\sum_{\tau_{j} \in \mathbf{h i p}(s)}\left\{\left\lceil\frac{{ }_{s} R_{p}^{\mathrm{LO}}}{T_{j}}\right\rceil C_{j}(\mathrm{LO})\right\} \\
& +\sum_{\tau_{k} \in \mathbf{p s} \backslash\left\{\tau_{p}\right\}} \max _{\tau_{q} \in \mathbf{h i p}(s)}\left\{I_{k}^{q}\left({ }_{s} R_{p}^{\mathrm{LO}}, \mathrm{LO}\right)\right\}
\end{aligned}
$$




$$
\begin{aligned}
{ }_{s} R_{p}^{\mathrm{HI}}= & \left(2-U_{p}(\mathrm{HI})\right) U_{p}(\mathrm{HI}) T_{s} \\
& +\sum_{\tau_{j} \in \mathbf{h i p H}(s)}\left\{\left\lceil\frac{{ }_{s} R_{p}^{\mathrm{HI}}}{T_{j}}\right\rceil C_{j}(\mathrm{HI})\right\} \\
& +\sum_{\tau_{k} \in \mathbf{p s} \backslash\left\{\tau_{p}\right\}} \max _{\tau_{q} \in \mathbf{h i p H}(s)}\left\{I_{k}^{q}\left({ }_{s} R_{p}^{\mathrm{HI}}, \mathrm{HI}\right)\right\}
\end{aligned}
$$

As with the traditional response time analysis of PIBS, its deadline is the same as that of its corresponding Sporadic Server $\tau_{s}$. Therefore, the above analysis must be applied to all Sporadic Servers associated with a PIBS.

\section{B. IO-AMC-rtb}

The techniques described in Section III are used for the IO-AMC-rtb analysis. Specifically, LO-criticality PIBS are allowed to continue execution in the HI-criticality mode. For a Sporadic Server task the IO-AMC-rtb equation is:

$$
\begin{aligned}
& R_{i}^{*}=C_{i}+\sum_{\tau_{j} \in \mathbf{h p H}(i)}\left\lceil\frac{R_{i}^{*}}{T_{j}}\right\rceil C_{j}(\mathrm{HI}) \\
&+\sum_{\tau_{j} \in \mathbf{h p L}(i)}\left\lceil\frac{R_{i}^{\mathrm{LO} *}}{T_{j}}\right\rceil C_{j}(\mathrm{LO}) \\
&+\sum_{\tau_{k} \in \mathbf{p s H}}\left\{\max _{\tau_{q} \in \mathbf{h i p}(i)} I_{k}^{q}\left(R_{i}^{*}, \mathrm{HI}\right)\right\} \\
&+\sum_{\tau_{k} \in \mathbf{p s L}}\left\{\max _{\tau_{q} \in \mathbf{h i p}(i)} I_{k}^{q}\left(R_{i}^{\mathrm{LO} *}, \mathrm{LO}\right)+\right. \\
&\left.\max _{\tau_{q^{\prime}} \in \mathbf{h i p H}(i)} I_{k}^{q^{\prime}}\left(R_{i}^{*}-R_{i}^{\mathrm{LO} *}, \mathrm{HI}\right)\right\}
\end{aligned}
$$

where psH and psL are the set of $\mathrm{HI}$ and LO-criticality PIBS respectively. The last summation in Equation 14 represents the maximum interference a LO-criticality PIBS can cause. Specifically, $I_{k}^{q}\left(R_{i}^{\mathrm{LO}}, \mathrm{LO}\right)$ represents the maximum interference the PIBS can cause before the mode change and $I_{k}^{q^{\prime}}\left(R_{i}^{*}-R_{i}^{\mathrm{LO}}, \mathrm{HI}\right)$ represents the total interference the PIBS can cause after the mode change. Again, the Sporadic Server that maximizes the interference is chosen for each PIBS.

The IO-AMC-rtb equation for a PIBS $\tau_{k}$ when assigned to Sporadic Server $\tau_{s}$ is:

$$
\begin{aligned}
{ }_{s} R_{p}^{*}= & \left(2-U_{p}(\mathrm{HI})\right) T_{s} U_{p}(\mathrm{HI}) \\
& +\sum_{\tau_{j} \in \mathbf{h i p H}(s)}\left\lceil\frac{{ }_{s} R_{p}^{*}}{T_{j}}\right] C_{j}(\mathrm{HI}) \\
& +\sum_{\tau_{j} \in \mathbf{h i p L}(s)}\left\lceil\frac{{ }_{s} R_{p}^{\mathrm{LO} *}}{T_{j}}\right\rceil C_{j}(\mathrm{LO}) \\
& +\sum_{\tau_{k} \in\left(\mathbf{p s H} \backslash\left\{\tau_{p}\right\}\right)}\left\{\max _{\tau_{q} \in \mathbf{h i p}(s)} I_{k}^{q}\left({ }_{s} R_{p}^{*}, \mathrm{HI}\right)\right\} \\
& +\sum_{\tau_{k} \in\left(\mathbf{p s L} \backslash\left\{\tau_{p}\right\}\right)}\left\{\max _{\tau_{q} \in \mathbf{h i p}(s)} I_{k}^{q}\left({ }_{s} R_{p}^{\mathrm{LO} *}, \mathrm{LO}\right)+\right. \\
& \left.\max _{\tau_{q^{\prime}} \in \mathbf{h i p H}(s)} I_{k}^{q^{\prime}}\left({ }_{s} R_{p}^{*}-{ }_{s} R_{p}^{\mathrm{LO} *}, \mathrm{HI}\right)\right\}
\end{aligned}
$$

Equation 15 differs from Equation 14 in the first term, and by the exclusion of $\tau_{p}$ from the set of PIBS. Similar to Equation 2] the response time analysis requires iterating over all HI-criticality Sporadic Servers that could be associated with the PIBS. This is because only the HI-criticality Sporadic Servers are of interest after the mode change.

As mentioned in Section III recent related work by Burns and Baruah [10] has extended the original AMC model to allow LO-criticality tasks to continue running after the mode to the HI-criticality mode. The derivation this work provided was used to allow LO-criticality PIBS to continue running after the mode change. This work can also be applied to allow LO-criticality Sporadic Servers to continue running in the HIcriticality mode. This derivation is similar to the one provided by Burns and Baruah but there are subtle differences due to the inclusion of PIBS.

First for Sporadic Servers during the mode change the new IO-AMC-rtb equation is:

$$
\begin{aligned}
R_{i}^{*}=C_{i} & +\sum_{\tau_{j} \in \mathbf{h p H}(i)}\left\lceil\frac{R_{i}^{*}}{T_{j}}\right\rceil C_{j}(\mathrm{HI}) \\
& +\sum_{\tau_{j} \in \mathbf{h p L}(i)}\left\lceil\frac{R_{i}^{\mathrm{LO} *}}{T_{j}}\right\rceil C_{j}(\mathrm{LO}) \\
& +\sum_{\tau_{j} \in \mathbf{h p L}(i)}\left(\left\lceil\frac{R_{i}^{*}}{T_{j}}\right\rceil-\left\lceil\frac{R_{i}^{\mathrm{LO} *}}{T_{j}}\right\rceil\right) C_{j}(\mathrm{HI}) \\
& +\sum_{\tau_{k} \in \mathbf{p s H}}\left\{\max _{\tau_{q} \in \mathbf{h i p}(i)} I_{k}^{q}\left(R_{i}^{*}, \mathrm{HI}\right)\right\} \\
& +\sum_{\tau_{k} \in \mathbf{p s L}}\left\{\max _{\tau_{q} \in \mathbf{h i p}(i)} I_{k}^{q}\left(R_{i}^{\mathrm{L} 0 *}, \mathrm{LO}\right)+\right. \\
& \left.\max _{\tau_{q^{\prime}} \in \mathbf{h i p}(i)} I_{k}^{q^{\prime}}\left(R_{i}^{*}-R_{i}^{\mathrm{LO} *}, \mathrm{HI}\right)\right\}
\end{aligned}
$$

In addition to the third term which is taken from Equation 9 Equation 16 differs from Equation 14 (where L0-criticality Sporadic Servers do not run in the HI-criticality mode) in that all Sporadic Servers of higher or equal priority must be considered when accounting for the interference from LOcriticality PIBS after the mode change. Specifically the hipH in the final term has changed to hip to reflect that fact that Sporadic Servers of all criticality levels run in the HI-criticality mode.

Equation 17 is the IO-AMC-rtb equation for PIBS when LO-criticality Sporadic Servers are allowed to run in the HIcriticality mode. Again the only differences are the inclusion of the interference caused by LO-criticality tasks after the mode change and changing the hipH to hip in the final term to account for the fact that all Sporadic Servers are capable of 
executing after the mode change.

$$
\begin{aligned}
{ }_{s} R_{p}^{*}= & \left(2-U_{p}(\mathrm{HI})\right) T_{s} U_{p}(\mathrm{HI}) \\
& +\sum_{\tau_{j} \in \mathbf{h i p H}(s)}\left\lceil\frac{{ }_{s} R_{p}^{*}}{T_{j}}\right\rceil C_{j}(\mathrm{HI}) \\
& +\sum_{\tau_{j} \in \mathbf{h i p L}(s)}\left\lceil\frac{{ }_{s} R_{p}^{\mathrm{LO} *}}{T_{j}}\right\rceil C_{j}(\mathrm{LO}) \\
& +\sum_{\tau_{j} \in \mathbf{h p L}(i)}\left(\left\lceil\frac{{ }_{s} R_{p}^{*}}{T_{j}}\right\rceil-\left\lceil\frac{{ }_{s} R_{p}^{\mathrm{LO} *}}{T_{j}}\right\rceil\right) C_{j}(\mathrm{HI}) \\
& +\sum_{\tau_{k} \in\left(\mathbf{p s H} \backslash\left\{\tau_{p}\right\}\right)}\left\{\max _{\tau_{q} \in \mathbf{h i p}(s)} I_{k}^{q}\left({ }_{s} R_{p}^{*}, \mathrm{HI}\right)\right\} \\
& +\sum_{\tau_{k} \in\left(\mathbf{p s L} \backslash\left\{\tau_{p}\right\}\right)}\left\{\max _{\tau_{q} \in \mathbf{h i p}(s)} I_{k}^{q}\left({ }_{s} R_{p}^{\mathrm{LO} *}, \mathrm{LO}\right)+\right. \\
& \left.\max _{\tau_{q^{\prime}} \in \mathbf{h i p}(s)} I_{k}^{q^{\prime}}\left({ }_{s} R_{p}^{*}-{ }_{s} R_{p}^{\mathrm{LO} *}, \mathrm{HI}\right)\right\}
\end{aligned}
$$

\section{IMPLEMENTATION}

Changes to the existing Quest scheduler to support IO-AMC scheduling include:

- Mapping Quest processes to a task and job model;

- Detecting when to change to HI-criticality mode;

- Adjusting Sporadic Server and PIBS replenishments.

Quest tasks are assigned to Sporadic Servers, and are similar to UNIX processes in that they run until an _exit system call is invoked. In comparison, real-time tasks in the IO-AMC model release a job at a specific rate and the job runs until completion. To accommodate the differences, a new sync system call was introduced. The sync function indicates the start and end of real-time jobs. A typical IO-AMC task has a setup phase, followed by a loop which repeatedly calls sync before starting the body of the next job.

A typical IO-AMC application will look similar to the procedure outlined in Algorithm 1.

\begin{tabular}{l}
\hline Algorithm 1 IO-AMC User Space Process \\
\hline procedure MAIN \\
// Setup Code \\
while TRUE do \\
sync () \\
// Job Code \\
end while \\
end procedure \\
\hline
\end{tabular}

An alternative approach would be to have one process repeatedly fork another process, with the newly forked process being the job for that period. This approach would not require the application developer to invoke the sync system call as the _exit system call could be used to inform the scheduler that the job is completed. This approach was not chosen however due to the extra overhead involved in the creation of a new process.

The first time sync is called, a mixed-criticality task will sleep until its Sporadic Server is replenished to full capacity.
The deadline will be set $T$ time units after the process wakes up. Subsequent calls to sync will have the process sleep up to the deadline, emulating the job being completed and waiting for the next job.

Quest bottom half threads are assigned to Priority Inheritance Bandwidth Preserving Servers, and follow the real-time task and job model. Bottom half threads are only woken up by the top halves, and at the end of their execution they notify the scheduler of their completion. Therefore, no changes needed to be made to the PIBS and bottom half threads.

The conditions for a mode change depend on whether a task is running a job or bottom half on a Sporadic Server or PIBS. A HI-criticality Sporadic Server initiates a mode change when it has depleted all replenishment items that are before the deadline. If this happens the Sporadic Server will not be able to run until after the deadline and therefore a mode change must occur. A HI-criticality PIBS causes a mode change when it has depleted its budget before completing the bottom half thread. In this case, the single replenishment for a PIBS will be at the deadline and therefore the PIBS will not be able to run until after its deadline unless a mode change occurred.

Finally, when the mode change occurs, the Sporadic Server and PIBS replenishment items must be adjusted to take into account the new or removed budget. For HI-criticality Sporadic Servers, the difference between $C(\mathrm{HI})$ and $C(\mathrm{LO})$ is added to the beginning of the first replenishment list item, if the item's replenishment time is equal to or less than the current time, or if the replenishment list is full. Otherwise, a new replenishment list item is inserted at the beginning with a replenishment time equal to the current time. A replenishment item $R$ has two properties, R.amt, which is the amount of budget replenished, and R.time, which is when the replenishment occurs. This is demonstrated in Algorithm 2

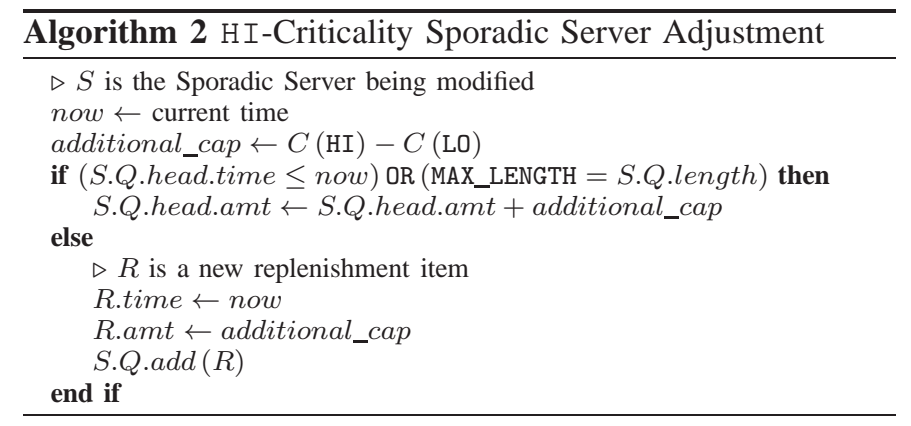

For LO-criticality Sporadic Servers, the adjustment algorithm is more complicated. If $C(\mathrm{LO})-C(\mathrm{HI})$ is less than or equal to the remaining budget for this period, i.e. before the deadline, then budget is removed from the replenishment list by moving backwards in time from the replenishment item right before the deadline. The head replenishment item must be treated differently if the Sporadic Server has used some of that budget at the time of a mode change. S.usage tracks how much has been used from the head replenishment item. If S.usage is greater than zero, a new replenishment item might be posted at the end of the queue with an amount equal 
to S.usage. If $C(\mathrm{LO})-C(\mathrm{HI})$ is greater than the remaining budget for this period then the Sporadic Server has run for more than its $C(\mathrm{HI})$ for this period. In this case all the available budget in the replenishment list is removed for this period and the difference is removed from the end of the replenishment list. Algorithm 3 contains the pseudocode for adjusting the budget of a LO-criticality Sporadic Server.

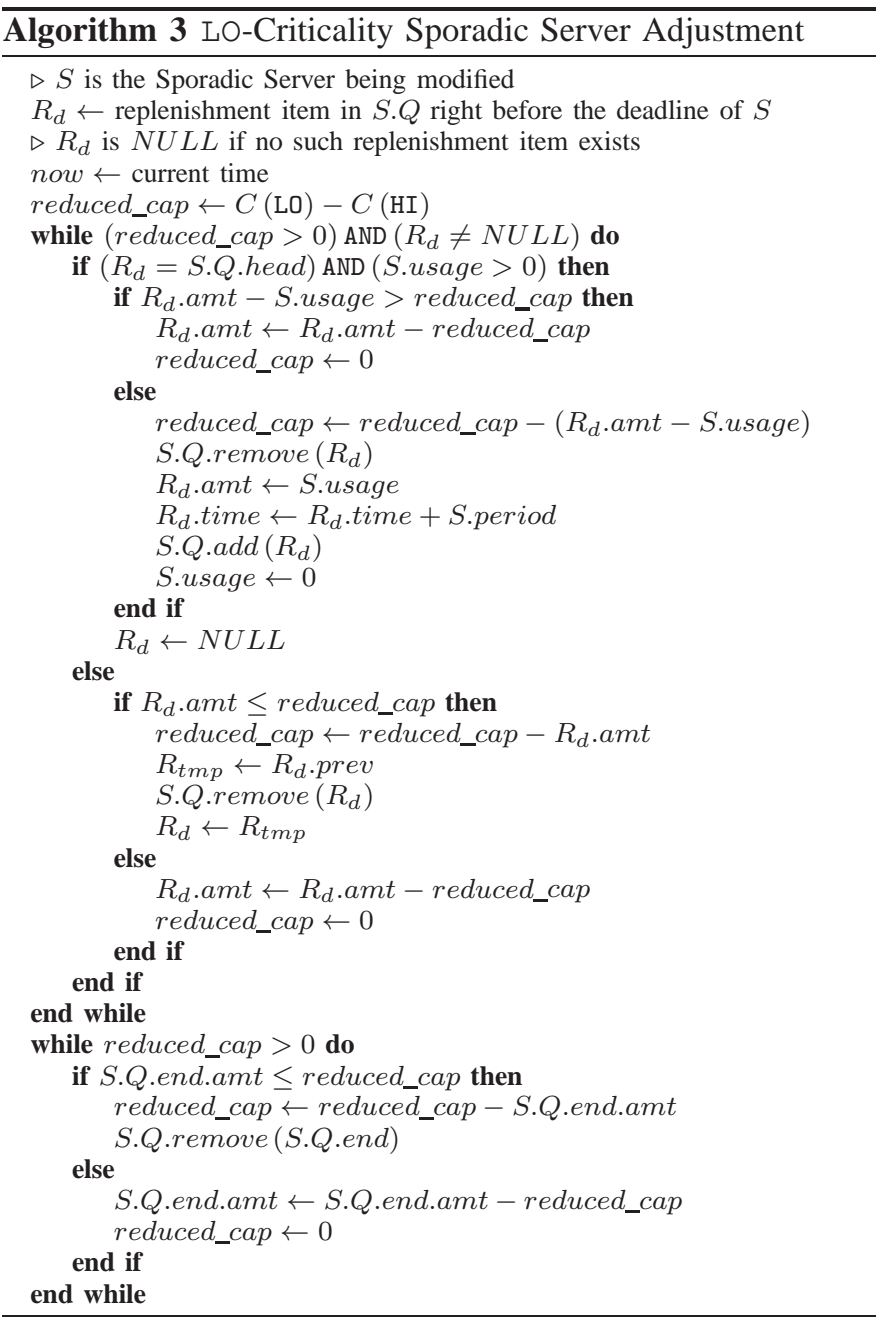

PIBS have a much simpler mode change algorithm because there is only one replenishment item to consider for each invocation of the server. Also, due to the aperiodicity of $\mathrm{I} / \mathrm{O}$ events, a deadline for a PIBS is calculated from when an $\mathrm{I} / \mathrm{O}$ event is initiated. This results in pessimistic analysis in Equations 14 and 15 . We have to assume that regardless of whether a PIBS is LO or HI-criticality, it causes the maximum interference possible in both the LO and HI modes. Therefore, both LO and HI PIBS can simply replenish their full budget at the time of a mode change.

\section{EVALUATION}

The experimental evaluation consists of two sections: 1) simulation based schedulability tests and 2) experiments conducted using the IO-AMC implementation in the Quest operating system. The simulation based schedulability tests show that a system of Sporadic Servers and PIBS have a similar but slightly lower schedulability than a system of just Sporadic Servers. This is due to the extra interference that can be caused by PIBS compared to Sporadic Servers. The Quest experiments show the benefits of PIBS compared to Sporadic Servers and how mixed-criticality can be used to control the bandwidth from I/O devices with different criticalities.

\section{A. Simulation Experiments}

In order to compare the proposed scheduling approaches, random task sets were generated with varying total utilizations. 500 task sets were generated for each utilization value ranging from 0.20 to 0.95 with 0.05 increments. Each task set was tested to see if it was schedulable under the different policies. Each PIBS was randomly assigned to a single Sporadic Server of the same criticality level. For systems comprising only Sporadic Servers, the PIBS were converted to Sporadic Servers of equivalent utilization and period 4 The parameters used to generate the task sets used are outlined in Table —

\begin{tabular}{|c|c|}
\hline Parameter & Value \\
\hline Number of Tasks & 20 (15 Main, 5 I/O) \\
\hline Criticality Factor & 2 \\
\hline Probability $L_{i}=\mathrm{HI}$ & 0.5 \\
\hline Period Range & $1-100$ \\
\hline I/O Total Utilization & 0.05 \\
\hline
\end{tabular}

TABLE I: Parameters Used to Generate Task Sets

The UUnifast algorithm [11] was used to generate the task sets. Task periods were generated with a log-uniform distribution. For the mixed-criticality experiments, $C_{i}(\mathrm{LO})=U_{i} / T_{i}$. If $L_{i}=\mathrm{HI}, C_{i}(\mathrm{HI})=\mathrm{CF} \times C_{i}(\mathrm{LO})$, where $\mathrm{CF}$ is the criticality factor. For our experiments, if $L_{i}=\mathrm{LO}, C_{i}(\mathrm{HI})=0$.

The following are the different types of schedulability tests that were used in the evaluation. This includes schedulability tests for mixed-criticality and traditional systems. leftmargin=*

- SS-rta - Sporadic Server response time analysis. Due to the nature of Sporadic Servers, this is the same as a periodic response time analysis.

- SS+PIBS-rta - Sporadic Server and PIBS response time analysis introduced in this paper. See Section 【I

- AMC-rtb - Adaptive Mixed-Criticality response time bound developed by Baruah et al. [2]. See Section III.

- IO-AMC-rtb - I/O Adaptive Mixed-Criticality response time bound developed in this paper. See Section IV.

- AMC UB - This is not a schedulability test but instead an upper bound for AMC. It consists of both the LO- and HI-criticality level steady states tests. See Section III for details.

- IO-AMC UB - This is not a schedulability test but instead an upper bound for IO-AMC. It consists of both the LO- and HI-criticality level steady states tests. See Section IV for details.

\footnotetext{
${ }^{4}$ The PIBS period was set equal to its corresponding Sporadic Server.
} 
1) SS+PIBS vs. SS-Only Simulations: Figure 7 contains the results of the response time analysis and event simulator for a system of Sporadic Servers and PIBS (SS+PIBS) compared to a system of only Sporadic Servers (SS-Only). As expected, a higher number of the Sporadic Server only task sets are schedulable using the response time analysis equations compared to the SS+PIBS response time analysis. This is due to the extra interference a PIBS can cause compared to a Sporadic Server of equivalent utilization and period.

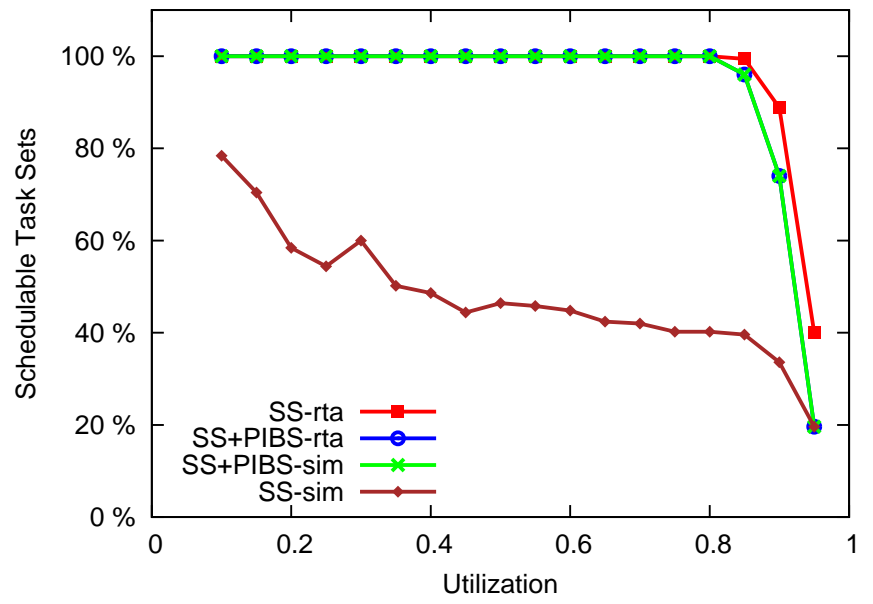

Fig. 7: Schedulability of SS+PIBS vs SS-Only

2) IO-AMC vs. AMC Simulations: In this section, IO-AMC is compared to an AMC system containing only Sporadic Servers under different mixed-criticality scenarios.

Figure 8 shows the response time analysis and simulation results when LO-criticality tasks do not run in the HI-criticality mode. Similar to Figure 7, AMC-rtb outperforms IO-AMCrtb. This is due to the fact that AMC-rtb is an extension of the traditional response time analysis and does not experience the extra interference caused by PIBS.

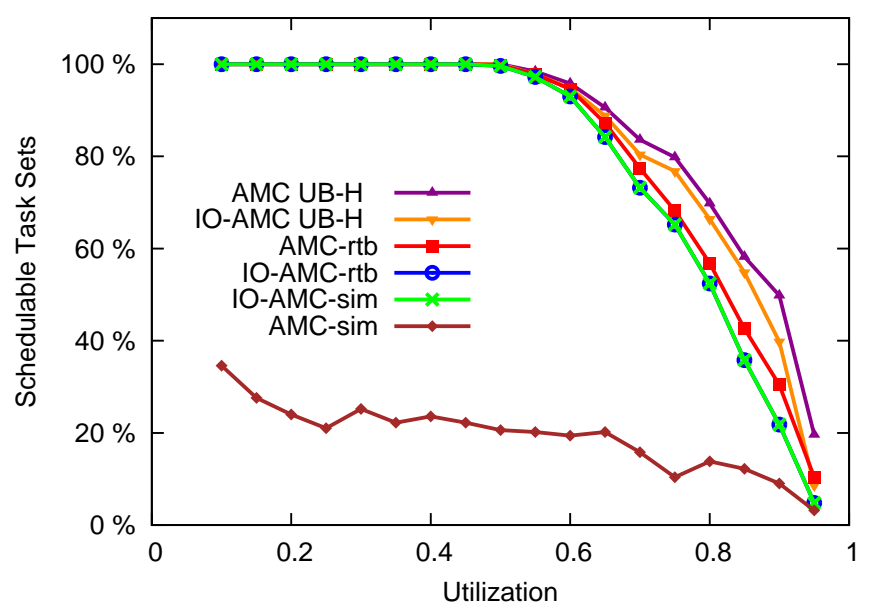

Fig. 8: Schedulability of IO-AMC vs AMC

We also varied task set parameters to identify their effects on schedulability. For each set of parameters $p$ in a given test $y$, we measured the weighted schedulability [12], which is defined as follows:

$$
W_{y}(p)=\sum_{\forall \tau}\left(u(\tau) \times S_{y}(\tau, p)\right) / \sum_{\forall \tau} u(\tau)
$$

where $S_{y}(\tau, p)$ is the binary result ( 0 or 1 ) of the schedulability test $y$ on task set $\tau$, and $u(\tau)$ is the total utilization. The weighted schedulability compresses a three-dimensional plot to two dimensions and places higher value on task sets with higher utilization.

Figures 9 , 10, and 11 show the results of varying the probability of a HI-criticality task, the criticality factor and the number of tasks respectively. In all scenarios, LO-criticality tasks do not run in the HI-criticality mode. As expected, the percentage of schedulable tasks for IO-AMC is slightly lower than the percentage for traditional AMC. This is again due to the slightly larger interference caused by a PIBS.

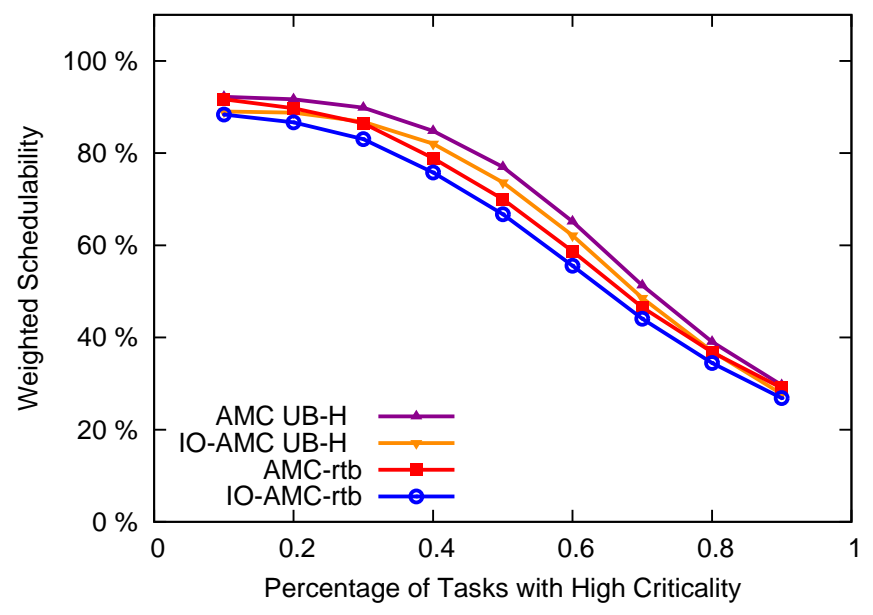

Fig. 9: Weighted Schedulability vs \% of HI-criticality Tasks

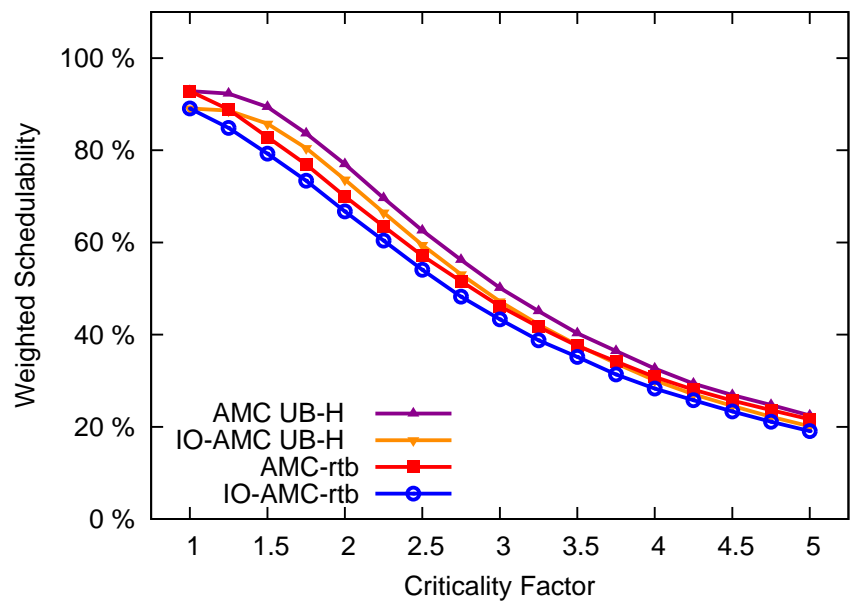

Fig. 10: Weighted Schedulability vs Criticality Factor 


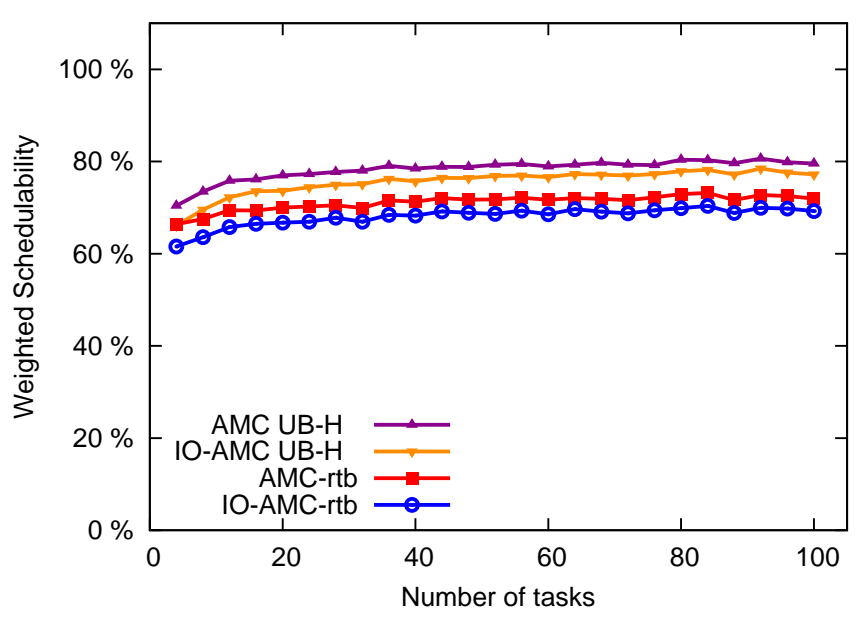

Fig. 11: Weighted Schedulability vs Number of Tasks

\section{B. Quest Experiments}

The above simulation results do not capture the practical costs of a system of servers for tasks and interrupt bottom halves. This section investigates the performance of our IOAMC policy in the Quest real-time system. We also study the effects of mode changes on I/O throughput for an application that collects streaming camera data. All experiments were run on a $3.10 \mathrm{GHz}$ Intel ${ }^{\circledR}$ Core i3-2100 CPU.

1) Scheduling Overhead: We studied the scheduling overheads for two different system implementations in Quest. In the first system, Sporadic Servers were used for both tasks and bottom halves (SS-Only). In the second system, Sporadic Servers were used for tasks, and PIBS were used to handle interrupt bottom halves (SS+PIBS). In both cases, a task set consisted of two application threads of different criticality levels assigned to two different Sporadic Servers, and one bottom half handler for interrupts from a USB camera. The first application thread read all the data available from the camera in a non-blocking manner and then busy-waited for its entire budget to simulate the time to process the data. The second application thread simply busy-waited for its entire budget, to simulate a CPU-bound task without any I/O requests. Both application threads consisted of a sequence of jobs. Each job was released once every server period or immediately after the completion of the previous job, depending on which was later. The experimental parameters are shown in Table $\Pi$.

\begin{tabular}{lccr} 
Task & $C(\mathrm{LO})$ or $U(\mathrm{LO})$ & $C(\mathrm{HI})$ or $U(\mathrm{HI})$ & $T$ \\
\hline $\begin{array}{l}\text { Application 1 } \\
\text { (HI-criticality) }\end{array}$ & $23 \mathrm{~ms}$ & $40 \mathrm{~ms}$ & $100 \mathrm{~ms}$ \\
\hline $\begin{array}{l}\text { Application 2 } \\
\text { (LO-criticality) }\end{array}$ & $10 \mathrm{~ms}$ & $1 \mathrm{~ms}$ & $100 \mathrm{~ms}$ \\
\hline Bottom Half (PIBS) & $U(\mathrm{LO})=1 \%$ & $U(\mathrm{HI})=2 \%$ & $100 \mathrm{~ms}$ \\
\hline Bottom Half (SS) & $1 \mathrm{~ms}$ & $2 \mathrm{~ms}$ & $100 \mathrm{~ms}$ \\
\hline
\end{tabular}

TABLE II: Quest Task Set Parameters for Scheduling Overhead

The processor's timestamp counter was recorded when each application finished its current job. Results are shown in
Figure 12 For SS+PIBS, each application completed its jobs at regular intervals. However, for SS-Only, the HI-criticality server for interrupts from the USB camera caused interference with the application tasks. This led to the HI-criticality task depleting its budget before finishing its job. This is due to the extra overhead added by a Sporadic Server handling the interrupt bottom half thread. Therefore, the system had to switch into the HI-criticality mode to ensure the HI-criticality task completed its job, sacrificing the performance of the LOcriticality task. This is depicted by the larger time between completed jobs in Figure 12. The SS+PIBS task set did not suffer from this problem due to the lower scheduling overhead caused by PIBS.

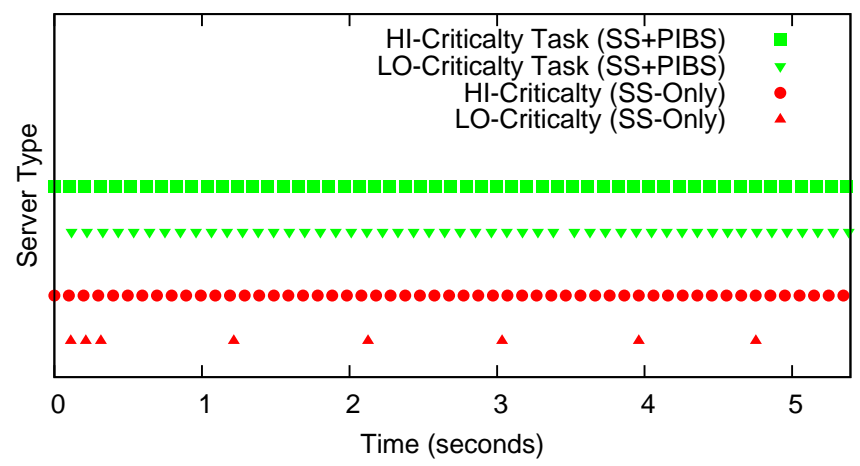

Fig. 12: Job Completion Times for SS+PIBS vs SS-Only

Figure 13 shows the additional overhead caused when Sporadic Servers are used for bottom half threads as opposed to PIBS. This higher scheduling overhead is the cause for the mode change in the previous experiment. Figure 13 depicts two different system configurations, one involving only a single camera and another involving two cameras. For each configuration, the scheduling overhead for both SS-Only and SS+PIBS was measured. For the single camera configuration, there is one HI-criticality task, one LO-criticality task, and one HI-criticality server (either PIBS or Sporadic Server) for the USB camera interrupt bottom half thread. The scheduling overhead for SS-Only is more erratic and higher than the system of Sporadic Servers and PIBS. The second configuration adds a LO-criticality camera with a $2 \%$ utilization in the LOcriticality mode, a $1 \%$ utilization in the HI-criticality mode, and a period of 100 microseconds when utilizing a Sporadic Server. Figure 13 shows that the scheduling overhead for an SS-Only system more than doubled, going from an average of $0.21 \%$ to $0.49 \%$, while an SS+PIBS system experienced only a small increase of $0.03 \%$.

2) Mode Change for I/O Device: As mentioned in Section IV] assigning criticality levels to bottom half interrupt handlers is akin to assigning criticality levels to the device associated with the bottom half. To test this assertion, two USB cameras were assigned different criticality levels and a mode change was caused during the execution of the task set. The task set consisted of two Sporadic Servers and two PIBS, as shown in Table III] 


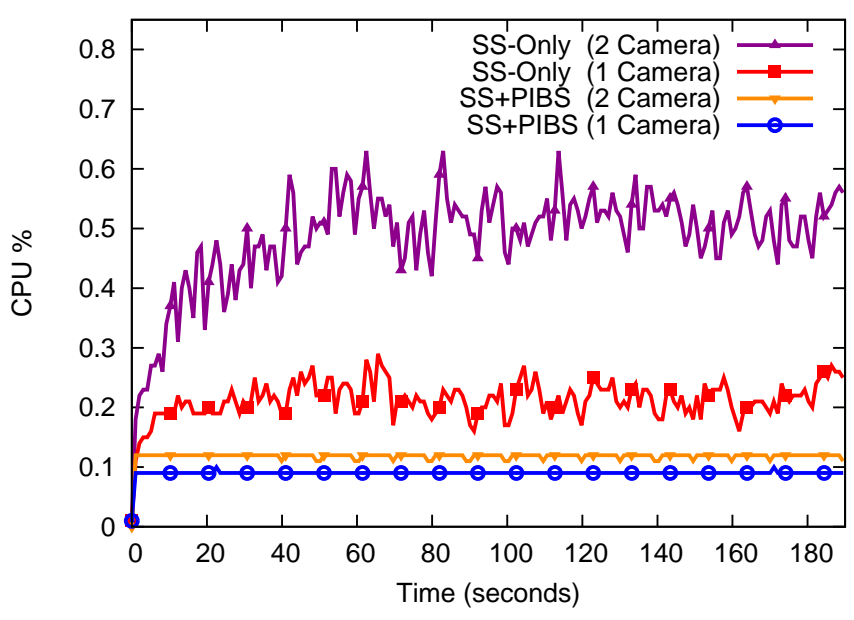

Fig. 13: Scheduling Overheads for SS-Only vs SS+PIBS

\begin{tabular}{lccr} 
Task & $C(\mathrm{LO})$ or $U(\mathrm{LO})$ & $C(\mathrm{HI})$ or $U(\mathrm{HI})$ & $T$ \\
\hline $\begin{array}{l}\text { Application 1 } \\
\text { (HI-criticality) }\end{array}$ & $25 \mathrm{~ms}$ & $40 \mathrm{~ms}$ & $100 \mathrm{~ms}$ \\
\hline $\begin{array}{l}\text { Application 2 } \\
\text { (LO-criticality) }\end{array}$ & $25 \mathrm{~ms}$ & $24 \mathrm{~ms}$ & $100 \mathrm{~ms}$ \\
\hline $\begin{array}{l}\text { Camera 1 - PIBS } \\
\text { (HI-criticality) }\end{array}$ & $U(\mathrm{LO})=0.1 \%$ & $U(\mathrm{HI})=1 \%$ & $100 \mathrm{~ms}$ \\
\hline $\begin{array}{l}\text { Camera 2 - PIBS } \\
\text { (LO-criticality) }\end{array}$ & $U(\mathrm{LO})=1 \%$ & $U(\mathrm{HI})=0.1 \%$ & $100 \mathrm{~ms}$ \\
\hline
\end{tabular}

TABLE III: Quest Task Set Parameters for I/O Device Mode Change

Figure 14 shows the camera data available at each data point. At approximately 30 seconds, a mode change occurs that causes Camera 1 to change from a utilization of $0.1 \%$ to $1 \%$, thereby increasing the amount of data received. Also at the time of the mode change, Camera 2's utilization switches from $1 \%$ to $0.1 \%$, causing a drop in received data. The variance for Camera 1 after the mode change is due to extra processing of the delayed data that is performed by the bottom half interrupt handler. Finally, Figure $[15$ shows the total data processed from each camera over time.

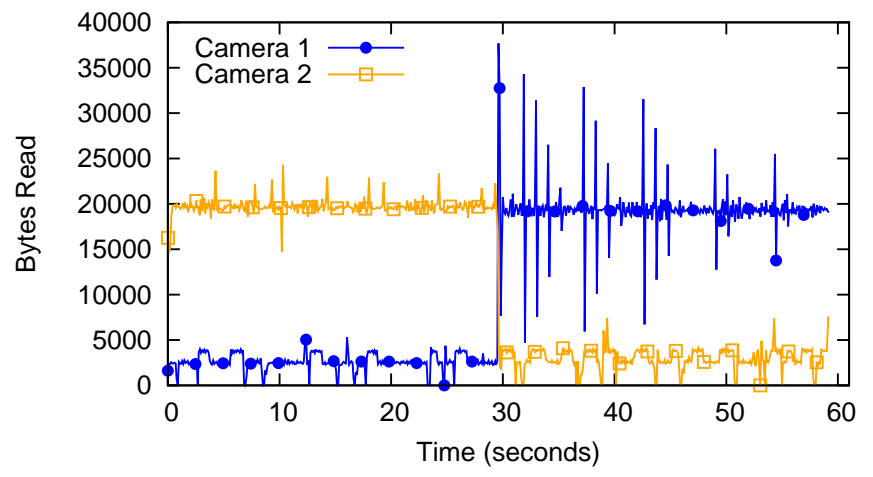

Fig. 14: Data From HI- and LO-criticality USB Cameras

\section{RELATED WORK}

The most relevant related work has already been mentioned in Sections $\Pi$ and IIII This section discusses additional work in the areas of mixed-criticality and I/O-aware scheduling.

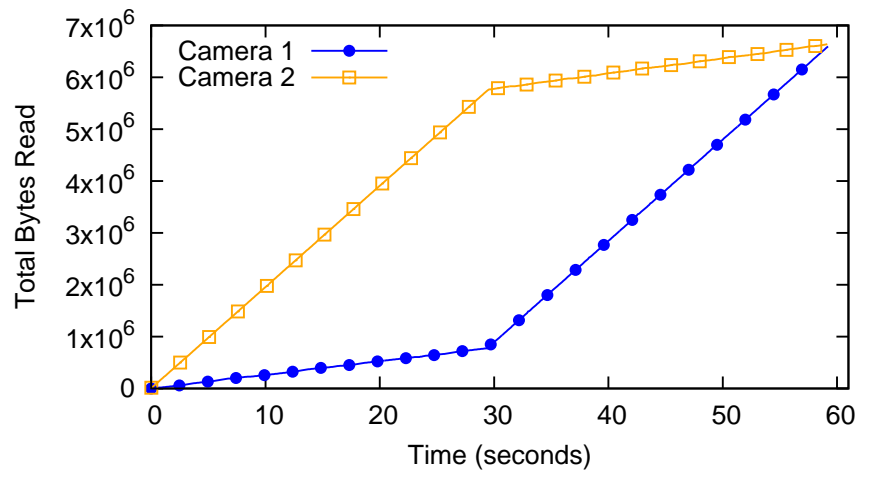

Fig. 15: Total Data Processed Over Time

In recent years, there have been many extensions to the Adaptive Mixed-Criticality model. For single core scheduling, Barauh, Burns and Davis extended their original AMC model to allow priorities to change [13]. Burns and Davis also introduced AMC-NPR (Non-Preemptive Region), which improved schedulability by permitting tasks to have a final non-preemptive region at the end of a job [14]. Fleming and Burns extended the AMC model to allow more than two criticality levels [15]. These variations on the mixed-critical model could be incorporated into the IO-AMC model.

$\mathrm{Li}$ and Barauh [16] combined the EDF-VD [17] singlecore mixed-criticality approach with fpEDF [18], to develop a multi-core mixed-criticality scheduling algorithm. Pathan also developed a multi-core fixed priority scheduling algorithm for mixed-criticality [19]. The approach adapted the original single-core AMC approach to a multi-core scheduling framework compatible with Audsley's algorithm [9]. The work by Pathan is more likely to be easily incorporated with the IOAMC model given that both approaches use fixed-priorities.

This work addresses the scheduling of and accounting of I/O events. Lewandowski et al. [20] investigated the use of sporadic servers to appropriately budget bottom half threads, as part of an Ethernet NIC device driver. Zhang and West developed a process-aware interrupt scheduling and accountability scheme in Linux, to integrate the management of tasks and I/O events [21]. A similar approach was also implemented in the LITMUS kernel for GPGPUs on multiprocessor systems [22].

\section{CONCLUSIONS}

This paper builds on our scheduling framework in the Quest real-time operating system, comprising a collection of Sporadic Servers for tasks and Priority Inheritance BandwidthPreserving Servers (PIBS) for interrupt handlers. We first show a response time analysis for a collection of Sporadic Servers and PIBS in a system without mixed criticality levels. We then extend the analysis to support an I/O Adaptive MixedCriticality (IO-AMC) model in a system comprising of tasks and interrupt handlers. Our IO-AMC response time bound considers a mode change to high-criticality when insufficient resources exist for either high-criticality tasks or interrupt handlers in low-criticality mode. The analysis considers the interference from low-criticality tasks and interrupt handlers before the mode change. 
Simulation results show that a system of only Sporadic Servers for both tasks and interrupt handlers has a higher theoretical number of schedulable task sets. However, in practice, using PIBS to handle interrupts is shown to be superior because of lower system overheads. This paper also shows experimental results in the Quest real-time operating system, where criticality levels are assigned to devices. This enables high criticality devices to gain more computational time when insufficient resources exist to service both high and low criticality tasks and interrupt bottom halves. In turn, this enables high criticality tasks that issue $\mathrm{I} / \mathrm{O}$ requests to be granted more CPU time to meet their deadlines.

The analysis in this paper assumes that tasks and I/O bottom half interrupt handlers are executed on separate servers that are independent of one another. In practice, a task may be blocked from execution until a pending $\mathrm{I} / \mathrm{O}$ request is completed. As long as the I/O request is handled within the time that a task is waiting for its server to have its budget replenished, and is therefore ineligible to run, then our analysis holds. Future work will consider more complex task models where I/O requests can lead to blocking delays that impact the execution of tasks.

\section{REFERENCES}

[1] S. Vestal, "Preemptive scheduling of multi-criticality systems with varying degrees of execution time assurance," in Real-Time Systems Symposium, 2007. RTSS 2007. 28th IEEE International, pp. 239-243, IEEE, 2007.

[2] S. K. Baruah, A. Burns, and R. I. Davis, "Response-time analysis for mixed criticality systems," in Real-Time Systems Symposium (RTSS), 2011 IEEE 32nd, pp. 34-43, IEEE, 2011.

[3] “Quest." http://www.QuestOS.org

[4] Y. Li, R. West, and E. Missimer, "A virtualized separation kernel for mixed criticality systems," in Proceedings of the 10th ACM SIGPLAN/SIGOPS international conference on Virtual execution environments, pp. 201-212, ACM, 2014.

[5] M. Danish, Y. Li, and R. West, "Virtual-CPU scheduling in the Quest operating system," in Real-Time and Embedded Technology and Applications Symposium (RTAS), 2011 17th IEEE, pp. 169-179, IEEE, 2011.

[6] B. Sprunt, Aperiodic task scheduling for real-time systems. $\mathrm{PhD}$ thesis, Carnegie Mellon University, 1990.
[7] C. L. Liu and J. W. Layland, "Scheduling algorithms for multiprogramming in a hard-real-time environment," Journal of the ACM (JACM), vol. 20, no. 1, pp. 46-61, 1973.

[8] M. Stanovich, T. P. Baker, A.-I. Wang, and M. G. Harbour, "Defects of the posix sporadic server and how to correct them," in Real-Time and Embedded Technology and Applications Symposium (RTAS), 2010 16th IEEE, pp. 35-45, IEEE, 2010.

[9] N. C. Audsley, "On priority assignment in fixed priority scheduling," Information Processing Letters, vol. 79, no. 1, pp. 39-44, 2001.

[10] A. Burns and S. Baruah, "Towards a more practical model for mixed criticality systems," in Workshop on Mixed-Criticality Systems (colocated with RTSS), 2013.

[11] E. Bini and G. C. Buttazzo, "Measuring the performance of schedulability tests," Journal of Real-Time Systems, vol. 30, no. 1-2, pp. 129-154, 2005.

[12] A. Bastoni, B. Brandenburg, and J. Anderson, "Cache-related preemption and migration delays: Empirical approximation and impact on schedulability," Proceedings of OSPERT, pp. 33-44, 2010.

[13] S. Baruah, A. Burns, and R. Davis, "An extended fixed priority scheme for mixed criticality systems," Proc. ReTiMiCS, RTCSA, pp. 18-24, 2013.

[14] A. Burns and R. Davis, "Adaptive mixed criticality scheduling with deferred preemption," in Real-Time Systems Symposium (RTSS), 2014 IEEE, pp. 21-30, IEEE, 2014.

[15] T. Fleming and A. Burns, "Extending mixed criticality scheduling," in Workshop on Mixed-Criticality Systems (colocated with RTSS), 2013.

[16] H. Li and S. Baruah, "Global mixed-criticality scheduling on multiprocessors," in Proceedings of the 2012 24th Euromicro Conference on Real-Time Systems, ECRTS, vol. 12, 2012.

[17] S. K. Baruah, V. Bonifaci, G. DAngelo, A. Marchetti-Spaccamela, S. Van Der Ster, and L. Stougie, "Mixed-criticality scheduling of sporadic task systems," in Algorithms-ESA 2011, pp. 555-566, Springer, 2011.

[18] S. K. Baruah, "Optimal utilization bounds for the fixed-priority scheduling of periodic task systems on identical multiprocessors," Computers, IEEE Transactions on, vol. 53, no. 6, pp. 781-784, 2004.

[19] R. M. Pathan, "Schedulability analysis of mixed-criticality systems on multiprocessors," in Real-Time Systems (ECRTS), 2012 24th Euromicro Conference on, pp. 309-320, IEEE, 2012.

[20] M. Lewandowski, M. J. Stanovich, T. P. Baker, K. Gopalan, and A.I. Wang, "Modeling device driver effects in real-time schedulability analysis: Study of a network driver," in Real Time and Embedded Technology and Applications Symposium, 2007. RTAS'07. 13th IEEE, pp. 57-68, IEEE, 2007.

[21] Y. Zhang and R. West, "Process-aware interrupt scheduling and accounting," in Real-Time Systems Symposium, 2006. RTSS'06. 27th IEEE International, pp. 191-201, IEEE, 2006.

[22] G. A. Elliott and J. H. Anderson, "Robust real-time multiprocessor interrupt handling motivated by gpus," in Real-Time Systems (ECRTS), 2012 24th Euromicro Conference on, pp. 267-276, IEEE, 2012. 